\title{
Blending Methodology of Linear Parameter Varying Control Synthesis of F-16 Aircraft System
}

\author{
Jong-Yeob Shin* \\ NASA Langley Research Center, Hampton, Virginia 23681 \\ Gary J. Balas ${ }^{\dagger}$ \\ University of Minnesota, Minneapolis, Minnesota 55455 \\ and \\ M. Alpay Kaya \\ University of California, Berkeley, Berkeley, California 94720
}

\begin{abstract}
The design of a linear parameter varying (LPV) controller for the F-16 longitudinal axes over the entire flight envelope, using a blending methodology that lets an LPV controller preserve regional optimal solutions over each parameter subset and reduces computational costs for synthesizing an LPV controller, is presented. Three blending LPV controller synthesis methodologies are applied to control F-16 longitudinal axes. By the use of a function substitution method, a quasi-LPV model of the F-16 longitudinal axes is constructed from the nonlinear equations of motion over the entire flight envelope, including nontrim regions, to facilitate synthesis of LPV controllers for the F-16 aircraft. The nonlinear simulations of the blended LPV controller show that the desired performance and robustness objectives are achieved across all altitude variations.
\end{abstract}

$C_{m_{0}}$
$C m_{q}$
$C_{X}, C_{Z}$
$C_{X_{q}}, C_{Z_{q}}$
$\bar{c}, S$
$g$
$m, I_{y y}$
$V, \bar{q}$
$X_{\text {a.c. }}, X_{\text {c.g. }}$
$\alpha, q$
$\delta_{e}, T$
$\theta, \gamma$

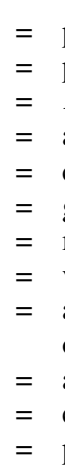

\section{Nomenclature}

pitch moment aerodynamic coefficient pitch moment aerodynamic stability derivative $X$ and $Z$ force aerodynamic coefficients aerodynamic stability derivatives chord length, $\mathrm{ft}$, and reference area, $\mathrm{ft}^{2}$ gravity constant, $\mathrm{ft} / \mathrm{s}^{2}$ mass, slug, and inertial moments, slug $\cdot \mathrm{ft}^{2}$ velocity, $\mathrm{ft} / \mathrm{s}$, and dynamic pressure, $\mathrm{psi}$ aerodynamic center position, $\mathrm{ft}$, and center of gravity position, $\mathrm{ft}$

angle of attack, rad, and pitch rate, $\mathrm{rad} / \mathrm{s}$

elevator deflection, rad, and thrust, $l b$

pitch angle, rad, and flight-path angle, rad

\section{Introduction}

$\mathbf{E}$ XTENSIVE research over the last 10 years has focused on developing analysis and synthesis techniques for gain-scheduled controllersfor linear parametervarying(LPV) systems. ${ }^{1-6}$ In Refs. 1 and 2, conditions are given that guarantee stability, robustness, and performanceproperties of the global gain-scheduleddesigns. Recent theoretical developments have produced methods for synthesizing gain-scheduling controllers for LPV systems, which guarantee a level of robust stability and performance across scheduling parameter spaces. $^{4-6}$ In Ref. 5, LPV control synthesis methods have also been developed using parameter-dependentLyapunov functions to lead to a less conservativeresult. This gain-scheduling approach has been successfully applied to synthesize controllers for pitch-axis

Presented as Paper 2001-4040 at the AIAA Guidance, Navigation, and Control Conference, Montreal, QC, Canada, 6-9 August 2001; received 23 October 2001; revision received 9 April 2002; accepted for publication 10 June 2002. Copyright (C) 2002 by the authors. Published by the American Institute of Aeronautics and Astronautics, Inc., with permission. Copies of this paper may be made for personal or internal use, on condition that the copier pay the $\$ 10.00$ per-copy fee to the Copyright Clearance Center, Inc., 222 Rosewood Drive, Danvers, MA 01923; include the code 0731-5090/02 $\$ 10.00$ in correspondence with the CCC.

* Staff Scientist, ICASE. Member AIAA.

${ }^{\dagger}$ Professor, Department of Aerospace Engineering and Mechanics. Member AIAA.

${ }^{\sharp}$ Graduate Research Assistant, Department of Mechanical Engineering. missile autopilots, ${ }^{7,8}$ F-14 aircraft lateral-directional axis during powered approach, ${ }^{9,10}$ and turbofan engines..$^{11,12}$

One of the potential difficulties in practical uses of the LPV synthesis methodology with parameter-dependentLyapunov functions is that the complexity of a linear matrix inequality (LMI) optimization problemincreases exponentially with the number of scheduling parameters and the number of grid points over a scheduling parameter space. One approach to facilitate practical use of LPV synthesis methodology, the "blending approach,"has been discussedin Refs. 6 and 13. This approach to control design partitions the entire parameter space into overlapping small subspaces. An LPV controller is synthesized for each small region. These regional controllers are blended into a single LPV controller for the entire parameter space. The blended LPV controllerpreserves the performancelevel of these regional controllers over each small region. In this paper, this blending approach is applied to control F-16 longitudinal axes over the entire flight envelope.

To synthesize an LPV controller for an F-16 aircraft, an LPV model of the aircraft dynamics is required. Conventionalapproaches to generate an LPV model of an aircraft are based on Jacobian linearization at trim points or a change of state coordinates ${ }^{8}$ to reduce the nonlinearity of aircraft dynamics. The LPV models constructed by both approaches can present aircraft dynamics at trim conditions. However, the models cannot represent aircraft dynamics at nontrim conditions. Instead of using Jacobian linearization or state transformation, the nonlinear terms of aircraft dynamics can be substituted for other functions in quasi-LPV form. ${ }^{14,15}$ This function substitution approach can be applied for both trim and nontrim conditions. The approach has been used in generating a quasi-LPV model of a generic missile. ${ }^{14,15}$ In this paper, a quasi-LPV model of F-16 longitudinal axes is provided over the entire flight envelope, including nontrim regions, using a function substitution approach.

In the second section, a brief summary of conventionalLPV controller synthesis used in this paper is presented to emphasize the complexity of the LMI optimizations. In the third section, three blending LPV control synthesis methodologies are presented. Development of a quasi-LPV model of F-16 longitudinal axes is presented in the fourth section. In the fifth section, formulation of the LPV control problem and blending two LPV controllers of the F-16 aircraftare presented. Nonlinearsimulations of the closed-loop system with the blended controller are presented in the sixth section, and this paper concludes with a brief summary in the seventh section. 


\section{LPV Control Synthesis}

In this section, a conventional LPV control synthesis using parameter-dependent Lyapunov functions ${ }^{5}$ is briefly described. Consider a generalized linear open-loop system as functions of parameters $\rho(t) \in \mathcal{P}$. For a compact subset $\mathcal{P} \subset \mathcal{R}^{s}$, the parameter variation set denotes the set of all piecewise continuous functions mapping $R$ (time) into $\mathcal{P}$ with a finite number of discontinuities in any interval, where $s$ is the number of parameters. An LPV openloop system can be written as

$$
\left[\begin{array}{c}
\dot{x}(t) \\
e(t) \\
y(t)
\end{array}\right]=\left[\begin{array}{ccc}
A(\rho(t)) & B_{1}(\rho(t)) & B_{2}(\rho(t)) \\
C_{1}(\rho(t)) & 0 & D_{12}(\rho(t)) \\
C_{2}(\rho(t)) & D_{21}(\rho(t)) & 0
\end{array}\right]\left[\begin{array}{c}
x(t) \\
d(t) \\
u(t)
\end{array}\right]
$$

where $y(t), e(t), d(t)$, and $u(t)$ are measurements, errors, disturbances, and control signals. Hereafter, $\rho$ denotes $\rho(t)$. The induced $\mathcal{L}_{2}$ norm of $d$ to $e$ is defined as

$$
\sup _{\rho \in \mathcal{P}, d \in \mathcal{L}_{2},\|d\|_{2} \neq 0} \frac{\|e\|_{2}}{\|d\|_{2}}
$$

Suppose there is an LPV outputfeedback controller $K(\rho)$ that stabilizes the closed-loopsystem exponentially and makes the induced$\mathcal{L}_{2}$ norm of $d$ to $e$ less than $\gamma$. An LPV controller $K(\rho)$ can be constructed from solutions of $X(\rho)$ and $Y(\rho)$ of the following optimization problem ${ }^{5}$ :

$$
\min _{X, Y \in \mathcal{R}^{n \times n}} \gamma
$$

subject to where matrices $Q(\boldsymbol{\rho}), F(\boldsymbol{\rho}), L(\boldsymbol{\rho})$, and $M(\boldsymbol{\rho}, \dot{\boldsymbol{\rho}})$ are defined as

$$
\begin{aligned}
& Q(\rho)=Y(\rho)-\gamma^{-2} X^{-1}(\rho) \\
& F(\rho)=-\left[B_{2}^{T}(\rho) X^{-1}(\rho)+D_{12}^{T}(\rho) C_{1}(\rho)\right] \\
& L(\rho)=-\left[Y^{-1}(\rho) C_{2}^{T}(\rho)+B_{1}(\rho) D_{21}^{T}(\rho)\right] \\
& M(\rho, \dot{\rho})=H(\rho, \dot{\rho})+\gamma^{2} Q(\rho)\left[-Q^{-1}(\rho) Y(\rho) L(\rho) D_{21}(\rho)\right. \\
& \left.\quad-B_{1}(\rho)\right] B_{1}^{T}(\rho) X^{-1}(\rho)
\end{aligned}
$$

Matrix $H(\rho, \dot{\rho})$ is defined as

$$
\begin{gathered}
H(\boldsymbol{\rho}, \dot{\boldsymbol{\rho}})=-\left[X^{-1}(\boldsymbol{\rho}) A_{F}(\boldsymbol{\rho})+A_{F}(\boldsymbol{\rho})^{T} X^{-1}(\boldsymbol{\rho})+\sum_{i=1}^{s}\left(\dot{\rho}_{i} \frac{\partial X^{-1}}{\partial \rho_{i}}\right)\right. \\
\left.+C_{F}^{T}(\boldsymbol{\rho}) C_{F}(\boldsymbol{\rho})+\gamma^{-2} X^{-1}(\boldsymbol{\rho}) B_{1}(\boldsymbol{\rho}) B_{1}(\boldsymbol{\rho})^{T} X^{-1}(\boldsymbol{\rho})\right]
\end{gathered}
$$

with $\quad A_{F}(\rho)=A(\rho)+B_{2}(\rho) F(\rho) \quad$ and $\quad C_{F}(\rho)=C_{1}(\rho)+$ $D_{12}(\rho) F(\rho)$. The closed-loop system with the controller $K(\rho)$ is exponentially stable, and the induced $-\mathcal{L}_{2}$ norm is less than $\gamma$. The proof can be found in Ref. 5 .

To make the optimization problem of Eq. (2) computationally tractable, the scheduling parameters $\rho$ are discretized into grid points. Thus, an infinite number of constraints are represented by a finite number of LMI constraints. Also, $X(\rho)$ and $Y(\rho)$ are represented by a finite number of basis functions $h_{i}(\rho)$ and $g_{i}(\rho)$ :

$$
\begin{aligned}
& {\left[\begin{array}{ccc}
X(\boldsymbol{\rho}) \hat{A}^{T}(\boldsymbol{\rho})+\hat{A}(\boldsymbol{\rho}) X(\boldsymbol{\rho})-\sum_{i=1}^{s}\left(\overline{\underline{v}}_{i} \frac{\partial X}{\partial \boldsymbol{\rho}_{i}}\right)-B_{2}(\boldsymbol{\rho}) B_{2}^{T}(\boldsymbol{\rho}) & X(\boldsymbol{\rho}) C_{11}^{T}(\boldsymbol{\rho}) & \gamma^{-1} B_{1}(\boldsymbol{\rho}) \\
C_{11}(\boldsymbol{\rho}) X(\boldsymbol{\rho}) & -I_{n_{e_{1}}} & 0 \\
\gamma^{-1} B_{1}^{T}(\boldsymbol{\rho}) & 0 & -I_{n_{d}}
\end{array}\right]<0} \\
& {\left[\begin{array}{ccc}
\tilde{A}(\boldsymbol{\rho}) Y(\boldsymbol{\rho})+Y(\boldsymbol{\rho}) \tilde{A}^{T}(\boldsymbol{\rho})+\sum_{i=1}^{s}\left(\underline{\underline{v}}_{i} \frac{\partial Y}{\partial \boldsymbol{\rho}_{i}}\right)-C_{2}^{T}(\boldsymbol{\rho}) C_{2}(\boldsymbol{\rho}) & Y(\boldsymbol{\rho}) B_{11}(\boldsymbol{\rho}) & \gamma^{-1} C_{1}^{T}(\boldsymbol{\rho}) \\
B_{11}^{T}(\boldsymbol{\rho}) Y(\boldsymbol{\rho}) & -I_{n_{d_{1}}} & 0 \\
\gamma^{-1} C_{1}(\boldsymbol{\rho}) & 0 & -I_{n_{e}}
\end{array}\right]<0}
\end{aligned}
$$

$$
\left[\begin{array}{ll}
X(\rho) & \gamma^{-1} I_{n} \\
\gamma^{-1} I_{n} & Y(\rho)
\end{array}\right] \geq 0, \quad X(\rho)>0, \quad Y(\rho)>0
$$

where

$$
\hat{A}(\rho) \equiv A(\rho)-B_{2}(\rho) C_{12}(\rho), \quad \tilde{A}(\rho) \equiv A(\rho)-B_{12}(\rho) C_{2}(\rho)
$$

and $n$ is the number of states of the generalized open-loop system. The detailed definitions of matrices $C_{11}(\rho), C_{12}(\rho)$, and $B_{11}(\rho)$ can be found in Ref. 5. Note that

$$
\sum_{i=1}^{s} \underline{\underline{v}}_{i}
$$

indicates that every combination of $\underline{v}_{i}$ and $\bar{v}_{i}$ is included in the LMIs. The parameter rate $\dot{\rho}$ is bounded as $\underline{\nu}_{i} \leq \dot{\rho}_{i} \leq \bar{v}_{i}$.

A method to construct an LPV controller $K(\rho)$ from the solutions $X(\rho)$ and $Y(\rho)$ is taken from Ref. 5. An LPV controller is constructed as

$$
\begin{aligned}
& -\gamma^{-2} Q^{-1}(\boldsymbol{\rho}) M(\boldsymbol{\rho}, \dot{\rho}) \\
& B_{k}(\boldsymbol{\rho})=-Q^{-1}(\boldsymbol{\rho}) Y(\boldsymbol{\rho}) L(\boldsymbol{\rho}) \\
& C_{k}(\boldsymbol{\rho})=F(\boldsymbol{\rho}) \\
& D_{k}(\boldsymbol{\rho})=0
\end{aligned}
$$$$
A_{k}(\rho)=A(\rho)+B_{2}(\rho) F(\rho)+Q^{-1}(\rho) Y(\rho) L(\rho) C_{2}(\rho)
$$

$$
X(\boldsymbol{\rho})=\sum_{j=1}^{N_{x}} h_{j}(\boldsymbol{\rho}) X_{j}, \quad Y(\boldsymbol{\rho})=\sum_{i=1}^{N_{y}} g_{j}(\boldsymbol{\rho}) Y_{j}
$$

where $h_{j}(\boldsymbol{\rho})$ and $g_{j}(\boldsymbol{\rho})$ are continuously differentiable functions. The LMIs of Eqs. (3-5) are solved for all grid points of the scheduling parameters simultaneously. The size of the optimization problem is proportional to $2^{s+1} N_{g}$, where $s$ and $N_{g}$ are the number of scheduling parameters and total number of grid points over the scheduling parameter space. The number of decision variables are $\left(N_{x}+N_{y}\right)[n(n+1) / 2]$, where $N_{x}, N_{y}$, and $n$ are the order of basis functions of $X$ and $Y$ and the state order. Thus, computational time to solve the optimization problem of Eq. (2) is dependent on the number of grid points of scheduling parameters, the state order of a generalized open-loop system, and the basis function orders. The conventional LPV synthesis methodology may require expensive computationalcost (computationaltime and computermemory) when the number of scheduling parameters increases. Also, a conventionally designed LPV controller leads to a conservative result because the decision variables $X_{j}$ and $Y_{j}$ in Eq. (11) satisfy LMI constraints over the entire parameter set simultaneously.

\section{LPV Controller Blending Approach}

Instead of designing a single LPV controller for the entire parameter set at the same time in a conventional LPV synthesis, LPV controllers can be synthesized for parameter subsets, respectively, which are overlapped with each other. Then, these LPV controllers are blended into a single LPV controller over the entire parameter set to satisfy stability and to achieve the desired performance. The 
blended LPV controller can lead to less conservative results than the conventionally designed LPV controller because the decision variables of the LMI optimization problem satisfy the LMI constraint over each parameter subset, respectively. Also, the blended LPV controller preserves the regional optimal solutions. The main difficulty of this blending approach is how to combine the regional optimal solution over the overlapped parameter subset.

Consider the scheduling parameter vector $\rho \in \mathcal{P}$, which consists of subvectors $\boldsymbol{\rho}_{s} \in \mathcal{P}_{s}$ and $\boldsymbol{\rho}_{d} \in \mathcal{P}_{d}$. The parameter subset $\mathcal{P}_{s}$ can be partitioned into two subsets with the following conditions:

$$
\begin{gathered}
\mathcal{P}_{s_{\cap}}=\mathcal{P}_{s_{1}} \cap \mathcal{P}_{s_{2}} \\
\mathcal{P}_{s}=\mathcal{P}_{s_{1}} \cup \mathcal{P}_{s_{2}}
\end{gathered}
$$

Suppose that there exist LPV controllers $K_{1}$ and $K_{2}$ constructed from parameter-dependentLyapunov functions of a parameter subvector $\rho_{d}$, over each parameter subset $\mathcal{P}_{s_{i}} \cup \mathcal{P}_{d}, i=1,2$. Thus, $X_{i}$ and $Y_{i}$ are functions of $\boldsymbol{\rho}_{d}$ (not $\boldsymbol{\rho}_{s}$ ) over each parameter subset $\mathcal{P}_{s_{i}} \cup \mathcal{P}_{d}, i=1,2$. Also, the controller $K_{i}$ can stabilize the closedloop system and the induced- $\mathcal{L}_{2}$ norm of the closed-loop system is less than $\gamma_{i}$ over each parameter subset $\mathcal{P}_{s_{i}} \cup \mathcal{P}_{d}$.

When a scheduling parameter subvector $\rho_{s}$ is in the intersection subset $\mathcal{P}_{s_{n}}$, LMI solution matrices $X_{i}\left(\boldsymbol{\rho}_{d}\right)$ and $Y_{i}\left(\boldsymbol{\rho}_{d}\right)$ are combined into $X_{b}\left(\boldsymbol{\rho}_{s}, \boldsymbol{\rho}_{d}\right)$ and $Y_{b}\left(\boldsymbol{\rho}_{s}, \boldsymbol{\rho}_{d}\right)$, respectively. The blended matrices $X_{b}\left(\boldsymbol{\rho}_{s}, \boldsymbol{\rho}_{d}\right)$ and $Y_{b}\left(\boldsymbol{\rho}_{s}, \boldsymbol{\rho}_{d}\right)$ should be feasible solutions of the LMI constraints of Eqs. (3) and (4) over the parameter subset $\mathcal{P}_{s \cap} \cup \mathcal{P}_{d}$. When the parameter subvector $\rho_{s}$ is in the parameter subset $\mathcal{P}_{s i}-\mathcal{P}_{s \cap}$, then $X_{b}\left(\boldsymbol{\rho}_{s}, \boldsymbol{\rho}_{d}\right)$ and $Y_{b}\left(\boldsymbol{\rho}_{s}, \boldsymbol{\rho}_{d}\right)$ should be equal to $X_{i}\left(\rho_{d}\right)$ and $Y_{i}\left(\rho_{d}\right)$, respectively, and the partial derivatives of $\partial X_{b} / \partial \rho$ and $\partial Y_{b} / \partial \rho$ should be equal to $\partial X_{i} / \partial \rho$ and $\partial Y_{i} / \partial \rho$, respectively. There are three blending methods for $X_{b}$ and $Y_{b}$ to satisfy the feasibility condition and the boundary conditions.

For method 1, matrices $X_{b}\left(\boldsymbol{\rho}_{s}, \boldsymbol{\rho}_{d}\right)$ and $Y_{b}\left(\boldsymbol{\rho}_{s}, \boldsymbol{\rho}_{d}\right)$ can be written as

$$
\begin{aligned}
& X_{b}\left(\boldsymbol{\rho}_{s}, \boldsymbol{\rho}_{d}\right)=\sum_{i=1}^{2} b_{x_{i}}\left(\boldsymbol{\rho}_{s}\right) X_{i}\left(\boldsymbol{\rho}_{d}\right) \\
& Y_{b}\left(\boldsymbol{\rho}_{s}, \boldsymbol{\rho}_{d}\right)=\sum_{i=1}^{2} b_{y_{i}}\left(\boldsymbol{\rho}_{s}\right) Y_{i}\left(\boldsymbol{\rho}_{d}\right)
\end{aligned}
$$

where blending functions $b_{x_{i}}\left(\boldsymbol{\rho}_{s}\right)$ and $b_{y_{i}}\left(\boldsymbol{\rho}_{s}\right)$ are differentiable scalar functions. To satisfy the boundary conditions of $X_{b}$ and $Y_{b}$, blending functions $b_{x_{i}}\left(\boldsymbol{\rho}_{s}\right)$ and $b_{y_{i}}\left(\boldsymbol{\rho}_{s}\right)$ are defined as

$$
\begin{gathered}
b_{y_{i}}\left(\boldsymbol{\rho}_{s}\right)=b_{x_{i}}\left(\boldsymbol{\rho}_{s}\right)= \begin{cases}1, & \boldsymbol{\rho}_{s} \in \mathcal{P}_{s_{i}}-\mathcal{P}_{s_{\cap}} \\
0, & \boldsymbol{\rho}_{s} \in \mathcal{P}_{s}-\mathcal{P}_{s_{i}}\end{cases} \\
\frac{\partial b_{x_{i}}\left(\boldsymbol{\rho}_{s}\right)}{\partial \boldsymbol{\rho}_{s}}=0, \quad \frac{\partial b_{y_{i}}\left(\boldsymbol{\rho}_{s}\right)}{\partial \boldsymbol{\rho}_{s}}=0, \quad \boldsymbol{\rho}_{s} \in \mathcal{P}_{s_{i}}-\mathcal{P}_{s \cap}
\end{gathered}
$$

Suppose that the blending functions $b_{x_{i}}\left(\boldsymbol{\rho}_{s}\right)$ and $b_{y_{i}}\left(\boldsymbol{\rho}_{s}\right)$ satisfy the following conditions:

$$
\begin{array}{lll}
\sum_{i=1}^{2} b_{x_{i}}\left(\boldsymbol{\rho}_{s}\right)=1, & 0 \leq b_{x_{i}}\left(\boldsymbol{\rho}_{s}\right) \leq 1, & \boldsymbol{\rho}_{s} \in \mathcal{P}_{S_{\cap}} \\
\sum_{i=1}^{2} b_{y_{i}}\left(\boldsymbol{\rho}_{s}\right)=1, & 0 \leq b_{y_{i}}\left(\boldsymbol{\rho}_{s}\right) \leq 1, & \boldsymbol{\rho}_{s} \in \mathcal{P}_{s \cap}
\end{array}
$$

and $\gamma$ is chosen as $\max \left(\gamma_{1}, \gamma_{2}\right)$. Then, the following equations are satisfied because the LMIs of Eqs. (3) and (4) are convex with respect to $X$ and $Y$. Hereafter, $\rho$ dependence is omitted for convenience:

$$
\begin{gathered}
X_{b} \hat{A}^{T}+\hat{A} X_{b}-\sum_{i=1}^{s}\left(\overline{\underline{v}}_{i} \sum_{j=1}^{2} b_{x_{j}} \frac{\partial X_{j}}{\partial \rho_{i}}\right)-B_{2} B_{2}^{T} \\
+X_{b} C_{11}^{T} C_{11} X_{b}+\gamma^{-2} B_{1} B_{1}^{T}<0 \\
\tilde{A} Y_{b}+Y_{b} \tilde{A}^{T}+\sum_{i=1}^{s}\left(\bar{v}_{i} \sum_{j=1}^{2} b_{y_{j}} \frac{\partial Y_{j}}{\partial \rho_{i}}\right)-C_{2}^{T} C_{2} \\
+Y_{b} B_{11} B_{11}^{T} Y_{b}+\gamma^{-2} C_{1}^{T} C_{1}<0
\end{gathered}
$$

When the derivatives of blending functions $\partial b_{x_{i}}\left(\boldsymbol{\rho}_{s}\right) / \partial \boldsymbol{\rho}_{s}$ and $\partial b_{y_{i}}\left(\boldsymbol{\rho}_{s}\right) / \partial \boldsymbol{\rho}_{s}$ are small enough to satisfy the following inequalities,

$$
\begin{gathered}
\bar{\sigma}\left(\sum_{i=1}^{s}\left\{\underline{\underline{v}}_{i} \sum_{j=1}^{2} X_{j}(\boldsymbol{\rho}) \frac{\partial b_{x_{j}}\left(\boldsymbol{\rho}_{s}\right)}{\partial \boldsymbol{\rho}_{i}}\right\}\right)<\underline{\sigma}\left(M_{X}\right) \\
\bar{\sigma}\left(\sum_{i=1}^{s}\left\{\overline{\underline{v}}_{i} \sum_{j=1}^{2} Y_{j}(\boldsymbol{\rho}) \frac{\partial b_{y_{j}}\left(\boldsymbol{\rho}_{s}\right)}{\partial \boldsymbol{\rho}_{i}}\right\}\right)<\underline{\sigma}\left(M_{Y}\right)
\end{gathered}
$$

it is noted that the blended $X_{b}\left(\boldsymbol{\rho}_{s}, \boldsymbol{\rho}_{d}\right)$ and $Y_{b}\left(\boldsymbol{\rho}_{s}, \boldsymbol{\rho}_{d}\right)$ can be feasible solutions of LMIs of Eqs. (3) and (5) over the parameter subset $\mathcal{P}_{s \cap}$, where matrices $M_{X}$ and $M_{Y}$ are

$$
\begin{aligned}
M_{X} & \equiv X_{b} \hat{A}^{T}+\hat{A} X_{b}-\sum_{i=1}^{s}\left(\underline{\underline{v}}_{i} \sum_{j=1}^{2} b_{x_{j}} \frac{\partial X_{j}}{\partial \rho_{i}}\right)-B_{2} B_{2}^{T} \\
& +X_{b} C_{11}^{T} C_{11} X_{b}+\gamma^{-2} B_{1} B_{1}^{T} \\
M_{Y} & \equiv \tilde{A} Y_{b}+Y_{b} \tilde{A}^{T}+\sum_{i=1}^{s}\left(\overline{\underline{v}}_{i} \sum_{j=1}^{2} b_{y_{j}} \frac{\partial Y_{j}}{\partial \rho_{i}}\right)-C_{2}^{T} C_{2} \\
& +Y_{b} B_{11} B_{11}^{T} Y_{b}+\gamma^{-2} C_{1}^{T} C_{1}
\end{aligned}
$$

Here, $\bar{\sigma}$ and $\underline{\sigma}$ represent the maximum and the minimum singular values, respectively.

The procedure of designing an LPV controller over the entire parameter set using this blending method 1 is as follows:

1) Solve the LMI optimization of Eq. (2) for $X_{i}\left(\rho_{d}\right)$ and $Y_{i}\left(\rho_{d}\right)$ over two parameter subsets, respectively.

2) Define blending scalar functions $b_{x_{i}}\left(\boldsymbol{\rho}_{s}\right)$ and $b_{y_{i}}\left(\boldsymbol{\rho}_{s}\right)$ that satisfy the boundary conditions of Eqs. (16) and (17) and the derivative conditions of Eqs. (22) and (23). Note that the derivative conditions are sufficient for $X_{b}\left(\boldsymbol{\rho}_{s}, \boldsymbol{\rho}_{d}\right)$ and $Y_{b}\left(\boldsymbol{\rho}_{s}, \boldsymbol{\rho}_{d}\right)$ to be feasible solutions of the LMI constraints of Eqs. (3) and (4) over the parameter subset $\mathcal{P}_{s \cap} \cup \mathcal{P}_{d}$. A controller designer chooses candidate blending functions until the feasibility conditions of Eqs. (3) and (4) are satisfied.

3) Construct an LPV controller over the entire parameter set $\mathcal{P}_{s} \cup \mathcal{P}_{d}$ using Eqs. (7-10), from the calculated $X_{b}$ and $Y_{b}$ of Eqs. (14) and (15).

For method 2, an alternative way to calculate the blended matrices $X\left(\boldsymbol{\rho}_{s}, \boldsymbol{\rho}_{d}\right)$ and $Y\left(\boldsymbol{\rho}_{s}, \boldsymbol{\rho}_{d}\right)$ as feasible solutions of the LMIs of Eqs. (3-5) is adding extra LMIs in conventionalLPV synthesis with candidate blending functions. ${ }^{6}$ The extra LMIs are

$$
\begin{aligned}
& {\left[\begin{array}{ccc}
\sum_{j=1}^{2} b_{x_{j}} X_{j} \hat{A}^{T}+\hat{A} \sum_{j=1}^{2} b_{x_{j}} X_{j}+\sum_{i=1}^{s}\left(\bar{v}_{i} \frac{\partial}{\partial \rho_{i}} \sum_{j=1}^{2} b_{x_{j}} X_{j}\right)-B_{2} B_{2}^{T} & \sum_{j=1}^{2} b_{x_{j}} X_{j} C_{11}^{T} & \gamma^{-1} B_{1} \\
C_{11} \sum_{j=1}^{2} b_{x_{j}} X_{j} & -I_{n_{e_{1}}} & 0 \\
\gamma^{-1} B_{1}^{T} & 0 & -I_{n_{d}}
\end{array}\right]<0} \\
& {\left[\begin{array}{ccc}
\tilde{A} \sum_{j=1}^{2} b_{y_{j}} Y_{j}+\sum_{j=1}^{2} b_{y_{j}} Y_{j} \tilde{A}^{T}+\sum_{i=1}^{s}\left(\underline{\underline{v}}_{i} \frac{\partial}{\partial \rho_{i}} \sum_{j=1}^{2} b_{y_{j}} Y_{j}\right)-C_{2}^{T} C_{2} & \sum_{j=1}^{2} b_{y_{j}} Y_{j} B_{11} & \gamma^{-1} C_{1}^{T} \\
B_{11}^{T} \sum_{j=1}^{2} b_{y_{j}} Y_{j} & -I_{n_{d_{1}}} & 0 \\
\gamma^{-1} C_{1} & 0 & -I_{n_{e}}
\end{array}\right]<0}
\end{aligned}
$$


The procedure of designing an LPV controller over the entire parameter set using this blending method 2 is as follows:

1) Solve the LMI optimization of Eq. (2) for the solution matrices $X_{1}\left(\rho_{d}\right)$ and $Y_{1}\left(\rho_{d}\right)$ over one of the parameter subsets, denoted by $\mathcal{P}_{s_{1}} \cup \mathcal{P}_{d}$.

2) Define blending scalarfunctions $b_{x_{i}}\left(\boldsymbol{\rho}_{s}\right)$ and $b_{y_{i}}\left(\boldsymbol{\rho}_{s}\right)$ that satisfy the boundary conditions of Eqs. (16) and (17).

3) Solve the LMI optimization problem of Eq. (2) with the extra LMI constraints of Eqs. (24) and (25) for the solution matrices $X_{2}\left(\rho_{d}\right)$ and $Y_{2}\left(\rho_{d}\right)$ over the other parameter subset $\mathcal{P}_{s_{2}} \cup \mathcal{P}_{d}$.

4) Repeat step 3 of method 1.

The LMI optimization over the second parameter subset $\mathcal{P}_{s_{2}} \cup \mathcal{P}_{d}$ is related to the solution matrices $X_{1}$ and $Y_{1}$ over the first parameter subset $\mathcal{P}_{s_{1}} \cup \mathcal{P}_{d}$. Thus, defining the order of parameter subsets may affect the designed LPV controller over the entire parameter set.

Both methods 1 and 2 require appropriate blending functions to blend solution matrices over the parameter subset $\mathcal{P}_{s_{n}}$. Note that an LPV controller provided by the blending methods 1 and 2 changes depending on which blending functions are selected. It is unknown how this affects the closed-loop performance of the designed LPV controller.

For method 3, blending matrix functions are calculated to minimize the induced- $\mathcal{L}_{2}$ norm $\gamma$ over the parameter subset $\mathcal{P}_{s \cap} \cup \mathcal{P}_{d}$. The blended solution matrices $X_{b}$ and $Y_{b}$ are rewritten as

$$
\begin{gathered}
X_{b}\left(\boldsymbol{\rho}_{s}, \boldsymbol{\rho}_{d}\right)=\frac{1}{2}\left[X_{b_{1}}\left(\boldsymbol{\rho}_{s}\right) X_{1}\left(\boldsymbol{\rho}_{d}\right)+X_{1}\left(\boldsymbol{\rho}_{d}\right) X_{b_{1}}\left(\boldsymbol{\rho}_{s}\right)\right] \\
+\frac{1}{2}\left[X_{b_{2}}\left(\boldsymbol{\rho}_{s}\right) X_{2}\left(\boldsymbol{\rho}_{d}\right)+X_{2}\left(\boldsymbol{\rho}_{d}\right) X_{b_{2}}\left(\boldsymbol{\rho}_{s}\right)\right] \\
Y_{b}\left(\boldsymbol{\rho}_{s}, \boldsymbol{\rho}_{d}\right)=\frac{1}{2}\left[Y_{b_{1}}\left(\boldsymbol{\rho}_{s}\right) Y_{1}\left(\boldsymbol{\rho}_{d}\right)+Y_{1}\left(\boldsymbol{\rho}_{d}\right) Y_{b_{1}}\left(\boldsymbol{\rho}_{s}\right)\right] \\
\quad+\frac{1}{2}\left[Y_{b_{2}}\left(\boldsymbol{\rho}_{s}\right) Y_{2}\left(\boldsymbol{\rho}_{d}\right)+Y_{2}\left(\boldsymbol{\rho}_{d}\right) Y_{b_{2}}\left(\boldsymbol{\rho}_{s}\right)\right]
\end{gathered}
$$

where blending matrix functions $X_{b_{1}}\left(\boldsymbol{\rho}_{s}\right), X_{b_{2}}\left(\boldsymbol{\rho}_{s}\right), Y_{b_{1}}\left(\boldsymbol{\rho}_{s}\right)$, and $Y_{b_{2}}\left(\boldsymbol{\rho}_{s}\right)$ are differentiable symmetric matrix functions bounded over the parameter subset $\mathcal{P}_{s_{\cap}}$. To present the blending matrix functions, basis functions $g_{x_{k}}\left(\boldsymbol{\rho}_{s}\right), h_{x_{k}}\left(\boldsymbol{\rho}_{s}\right), g_{y_{k}}\left(\boldsymbol{\rho}_{s}\right)$, and $h_{y_{k}}\left(\boldsymbol{\rho}_{s}\right)$ for $X_{b_{1}}\left(\boldsymbol{\rho}_{s}\right)$, $X_{b_{2}}\left(\boldsymbol{\rho}_{s}\right), Y_{b_{1}}\left(\boldsymbol{\rho}_{s}\right)$, and $Y_{b_{2}}\left(\boldsymbol{\rho}_{s}\right)$ are introduced, respectively. To satisfy the boundary condition of blended matrices $X_{b}\left(\rho_{s}, \rho_{d}\right)$ and $Y_{b}\left(\boldsymbol{\rho}_{s}, \boldsymbol{\rho}_{d}\right)$, the $X_{b_{1}}\left(\boldsymbol{\rho}_{s}\right), X_{b_{2}}\left(\boldsymbol{\rho}_{s}\right), Y_{b_{1}}\left(\boldsymbol{\rho}_{s}\right)$, and $Y_{b_{2}}\left(\boldsymbol{\rho}_{s}\right)$ are defined as

$$
\begin{aligned}
& X_{b_{1}}\left(\boldsymbol{\rho}_{s}\right)=g_{x_{0}}\left(\boldsymbol{\rho}_{s}\right) I+\sum_{k=1}^{N_{x_{g}}} g_{x_{k}}\left(\boldsymbol{\rho}_{s}\right) X_{b_{1_{k}}} \\
& X_{b_{2}}\left(\boldsymbol{\rho}_{s}\right)=h_{x_{0}}\left(\boldsymbol{\rho}_{s}\right) I+\sum_{k=1}^{N_{x_{h}}} h_{x_{k}}\left(\boldsymbol{\rho}_{s}\right) X_{b_{2_{k}}} \\
& Y_{b_{1}}\left(\boldsymbol{\rho}_{s}\right)=g_{y_{0}}\left(\boldsymbol{\rho}_{s}\right) I+\sum_{k=1}^{N_{y_{g}}} g_{y_{k}}\left(\boldsymbol{\rho}_{s}\right) Y_{b_{1_{k}}} \\
& Y_{b_{2}}\left(\boldsymbol{\rho}_{s}\right)=h_{y_{0}}\left(\boldsymbol{\rho}_{s}\right) I+\sum_{k=1}^{N_{y_{h}}} h_{y_{k}}\left(\boldsymbol{\rho}_{s}\right) Y_{b_{2_{k}}}
\end{aligned}
$$

where

$$
\begin{array}{cc}
g_{x_{0}}\left(\boldsymbol{\rho}_{s}\right)=1, & h_{x_{k}}\left(\boldsymbol{\rho}_{s}\right)=0 \\
\frac{\partial g_{x_{k}}\left(\boldsymbol{\rho}_{s}\right)}{\partial \boldsymbol{\rho}_{s_{i}}}=\frac{\partial h_{x_{k}}\left(\boldsymbol{\rho}_{s}\right)}{\partial \boldsymbol{\rho}_{s_{i}}}=0, & \boldsymbol{\rho}_{s} \in \mathcal{P}_{s 1}-\mathcal{P}_{s \cap} \\
h_{x_{0}}\left(\boldsymbol{\rho}_{s}\right)=1, & g_{x_{k}}\left(\boldsymbol{\rho}_{s}\right)=0 \\
\frac{\partial g_{x_{k}}\left(\boldsymbol{\rho}_{s}\right)}{\partial \boldsymbol{\rho}_{s_{i}}}=\frac{\partial h_{x_{k}}\left(\boldsymbol{\rho}_{s}\right)}{\partial \boldsymbol{\rho}_{s_{i}}}=0, & \boldsymbol{\rho}_{s} \in \mathcal{P}_{s_{2}}-\mathcal{P}_{s \cap} \\
\frac{\partial g_{y_{k}}\left(\boldsymbol{\rho}_{s}\right)}{\partial \boldsymbol{\rho}_{s_{i}}}=\frac{\partial h_{y_{k}}\left(\boldsymbol{\rho}_{s}\right)}{\partial \boldsymbol{\rho}_{s_{i}}}=0, & h_{y_{k}}\left(\boldsymbol{\rho}_{s}\right)=0 \\
h_{y_{0}}\left(\boldsymbol{\rho}_{s}\right)=1, & \boldsymbol{\rho}_{s} \in \mathcal{P}_{s_{1}}-\mathcal{P}_{s_{n}} \\
& g_{y_{k}}\left(\boldsymbol{\rho}_{s}\right)=0
\end{array}
$$

$$
\frac{\partial g_{y k}\left(\boldsymbol{\rho}_{s}\right)}{\partial \boldsymbol{\rho}_{s_{i}}}=\frac{\partial h_{y_{k}}\left(\boldsymbol{\rho}_{s}\right)}{\partial \boldsymbol{\rho}_{s_{i}}}=0, \quad \boldsymbol{\rho}_{s} \in \mathcal{P}_{s_{2}}-\mathcal{P}_{s_{\cap}}
$$

Here, the basis functions are differentiableover the parameter subset $\mathcal{P}_{s \cap}$ and $X_{b_{1_{k}}}, X_{b_{2_{k}}}, Y_{b_{1_{k}}}$, and $Y_{b_{2_{k}}}$ are unknown constant matrices in $\mathcal{R}^{n \times n}$. The unknown constant matrices can be determined solving the following LMI optimization:

$$
\begin{aligned}
& \min _{X_{b_{1_{j}}}, X_{b_{2_{j}}}, Y_{b_{1} 1_{j}}, Y_{b_{2_{j}}} \in \mathcal{R}^{n \times n}} \gamma
\end{aligned}
$$

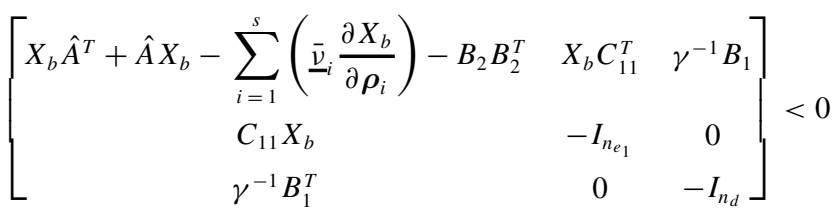

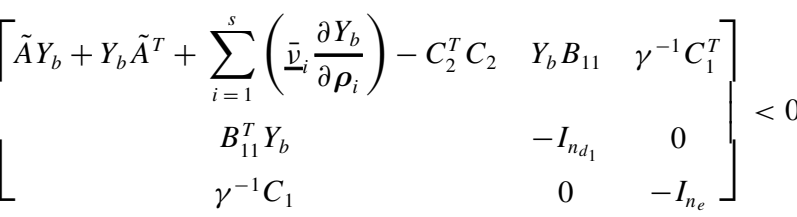

$$
\begin{aligned}
& {\left[\begin{array}{cc}
X_{b} & \gamma^{-1} I_{n} \\
\gamma^{-1} I_{n} & Y_{b}
\end{array}\right] \geq 0, \quad X_{b}>0, \quad Y_{b}>0}
\end{aligned}
$$

where

$$
\begin{aligned}
X_{b}= & g_{x_{0}} X_{1}+h_{x_{0}} X_{2}+0.5\left\{\sum_{j=1}^{N_{x_{g}}} g_{x_{j}}\left[X_{1} X_{b_{1_{j}}}+X_{b_{1 j}} X_{1}\right]\right. \\
& \left.+\sum_{k=1}^{N_{x_{h}}} h_{x_{k}}\left[X_{2} X_{b_{2_{k}}}+X_{b_{2_{k}}} X_{2}\right]\right\} \\
Y_{b}= & g_{y_{0}} Y_{1}+h_{y_{0}} Y_{2}+0.5\left\{\sum_{j=1}^{N_{y_{g}}} g_{y_{j}}\left[Y_{1} Y_{b_{1 j}}+Y_{b_{1_{j}}} Y_{1}\right]\right. \\
& \left.+\sum_{k=1}^{N_{y_{h}}} h_{y_{k}}\left[Y_{2} Y_{b_{2_{k}}}+Y_{b_{2_{k}}} Y_{2}\right]\right\}
\end{aligned}
$$

The procedure of designing an LPV controller over the entire parameter set using this blending method 3 is as follows:

1) Solve the LMI optimization of Eq. (2) for $X_{i}(\rho)$ and $Y_{i}(\rho)$ over each parameter subset.

2) Define basis functions for blending matrix functions in Eqs. (28) and (29), which satisfy the boundary conditions of Eq. (30) over each parameter subset.

3) Construct solution matrices $X$ and $Y$ over the entire parameter set using the solution matrices $X_{1}, X_{2}, Y_{1}, Y_{2}, X_{b}$, and $Y_{b}$, after solving the optimization of Eq. (31).

4) Construct an LPV controllerover the entire parameter set using Eqs. (7-10) based on solution matrices $X$ and $Y$ generated in step 3 .

There are three methods described to calculate the blended solution matrices $X_{b}\left(\boldsymbol{\rho}_{s}, \boldsymbol{\rho}_{d}\right)$ and $Y_{b}\left(\boldsymbol{\rho}_{s}, \boldsymbol{\rho}_{d}\right)$ that are feasible solutions of LMIs of Eqs. (3) and (4) over the parameter subset $\mathcal{P}_{s \cap} \cup \mathcal{P}_{d}$. Because all three methods keep $X_{1}$ and $Y_{1}$ over the parameter subset $\mathcal{P}_{s_{1}}-\mathcal{P}_{s_{\cap}}$, the blended LPV controllers generated by the three methods are equal to the regional LPV controller $K_{1}$ over the parameter subset $\mathcal{P}_{s_{1}}-\mathcal{P}_{s_{n}}$. Both methods 1 and 3 can also keep the regional LPV controller $K_{2}$ over the parameter subset $\mathcal{P}_{s_{2}}-\mathcal{P}_{s_{n}}$.

The disadvantage of method 1 is that choosing blending functions is performed in an ad hoc manner. However, method 1 is the fastest to synthesizean LPV controllerover the entire parameter set among the three blending methods. In method 2, the blending solution matrices $X_{b}$ and $Y_{b}$ are guaranteed to be feasible solutions over the parameter subset $\mathcal{P}_{s \cap}$. Method 2 requires solving the LMI optimization with 
the extra LMI constraints. In method 3, optimal blending matrix functionsare calculated to minimize the induced $-\mathcal{L}_{2}$ norm $\gamma$ over the parameter set $\mathcal{P}_{s \cap}$, based on basis functions defined by a controller designer. It is unknown how the basis functions affect the blending matrix functions and the LPV control design.

\section{Quasi-LPV Model of F-16 Longitudinal Axes}

In this section, a quasi-LPV model of F-16 longitudinal axes is presented. The full nonlinear equations of an F-16A aircraft are taken from Ref. 16. The nonlinear equations of F-16 longitudinal $\operatorname{axes}^{16}$ are

$$
\begin{aligned}
\dot{V}= & (\bar{q} S \cos \alpha / m)\left[C_{X}\left(\alpha, \delta_{e}\right)+(\bar{c} / 2 V) C_{X_{q}}(\alpha) q\right] \\
& +(\bar{q} S \sin \alpha / m)\left[C_{Z}\left(\alpha, \delta_{e}\right)+(\bar{c} / 2 V) C_{Z_{q}}(\alpha) q\right] \\
& -g \sin (\theta-\alpha)+(T / m) \cos \alpha
\end{aligned}
$$

$\dot{\alpha}=[g \cos (\theta-\alpha)] / V-(\sin \alpha / m V \cos \beta) T$

$$
\begin{aligned}
& +\left\{1+\left(\bar{q} S \bar{c} / 2 m V^{2}\right)\left[C_{Z_{q}}(\alpha) \cos \alpha-C_{X_{q}}(\alpha) \sin \alpha\right]\right\} q \\
& +(\bar{q} S / V)\left[C_{Z}\left(\alpha, \delta_{e}\right) \cos \alpha-C_{X}\left(\alpha, \delta_{e}\right) \sin \alpha\right]
\end{aligned}
$$

$\dot{q}=\left(\bar{q} S \bar{c} / 2 I_{y y} V\right)\left[\bar{c} C_{m_{q}}(\alpha)+\Delta C_{Z_{q}}(\alpha)\right] q$

$$
+\left(\bar{q} S \bar{c} / I_{y y}\right)\left[C_{m_{0}}\left(\alpha, \delta_{e}\right)+(\Delta / \bar{c}) C_{Z}\left(\alpha, \delta_{e}\right)\right]
$$

$\dot{\theta}=q$

where $\Delta=X_{\text {c.g. }}-X_{\text {a.c. }}$ Velocity, angle of attack, pitch rate, and pitch angle are the state variables, and thrust and elevator deflection are the control variables. The aerodynamic coefficients are lookup tables based on wind-tunnel data from NASA Langley Research Center wind-tunnel tests on an F-16 aircraft scaled model. ${ }^{17}$ The aerodynamic data were valid for a speed range of $100 \leq V \leq 900 \mathrm{ft} / \mathrm{s}$, an angle of attack range of $-10 \leq \alpha \leq 45 \mathrm{deg}$, and an altitude range of $5000 \leq h \leq 40,000 \mathrm{ft}$. These three parameters are scheduling parameters in the quasi-LPV model derived for the F-16 longitudinal axes. Note that $V$ and $\alpha$ are both scheduling parameters and states and that $h$ is a scheduling parameter that enters implicitly into the nonlinear dynamics.

Unfortunately, the control variable $\delta_{e}$ does not enter affinely in Eqs. (36-38). To derive a quasi-LPV model of F-16 longitudinal axes, it is necessary that all controls be in affine form. This is achieved by transforming $\left(\delta_{e}, T\right)$ into synthetic inputs $u_{s}=\left[\begin{array}{ll}u_{1} & u_{2}\end{array}\right]^{T}$. For details of the transformation,readers are referred to Ref. 13. For the F-16 quasi-LPV model, $\cos \theta$ and $\sin \theta$ are linearized about a trim value $\theta_{0}$. After tedious algebraic manipulations, Eqs. (36-39) are rewritten as

$$
\begin{gathered}
\dot{x}=A(V, \alpha, h) x+M(V, \alpha, h) u_{s}+f(V, \alpha, h) \\
x \equiv\left[\begin{array}{llll}
V & \alpha & q & \theta-\theta_{0}
\end{array}\right]^{T}
\end{gathered}
$$

where the elements of matrices $A \in \mathcal{R}^{4 \times 4}, \quad M \in \mathcal{R}^{4 \times 2}$, and $f \in \mathcal{R}^{4 \times 1}$ are given in Appendix A.

With the function substitution method, ${ }^{14,15}$ the nonlinear function $f(V, \alpha, h)$ can be decomposed into quasi-linear functions $G(V, \alpha, h)\left[\begin{array}{ll}V-V_{0} & \alpha-\alpha_{0}\end{array}\right]^{T}$ where $G$ is in $\mathcal{R}^{4 \times 2}$. Thus, a quasiLPV model of F-16 aircraft longitudinalaxes is provided. The details of function substitution are given in Appendix B. To compare simulation results of the nonlinear and the quasi-LPV model of the F-16 aircraft dynamics, several time sets of inputs $T$ and $\delta_{e}$ are predefined. In this paper, one example of time simulations is presented due to space limitations. For example, inputs are set as

$$
\delta_{e}=\delta_{e 0}, \quad T= \begin{cases}T_{0} \mathrm{lb}, & 0 \leq t \leq 1, \\ T_{0}-2000 \mathrm{lb}, & 1 \leq t \leq 11 \mathrm{~s}\end{cases}
$$

where $\delta_{e 0}$ and $T_{0}$ are a trim values. The time simulation results in Fig. 1 show that the time responses of the quasi-LPV and nonlinear models are almost matched to each other. Note that the quasi-LPV model provided by the function substitution method may change depending on which one trim point is selected. It is unknown how this affects the quasi-LPV model or the LPV control design.
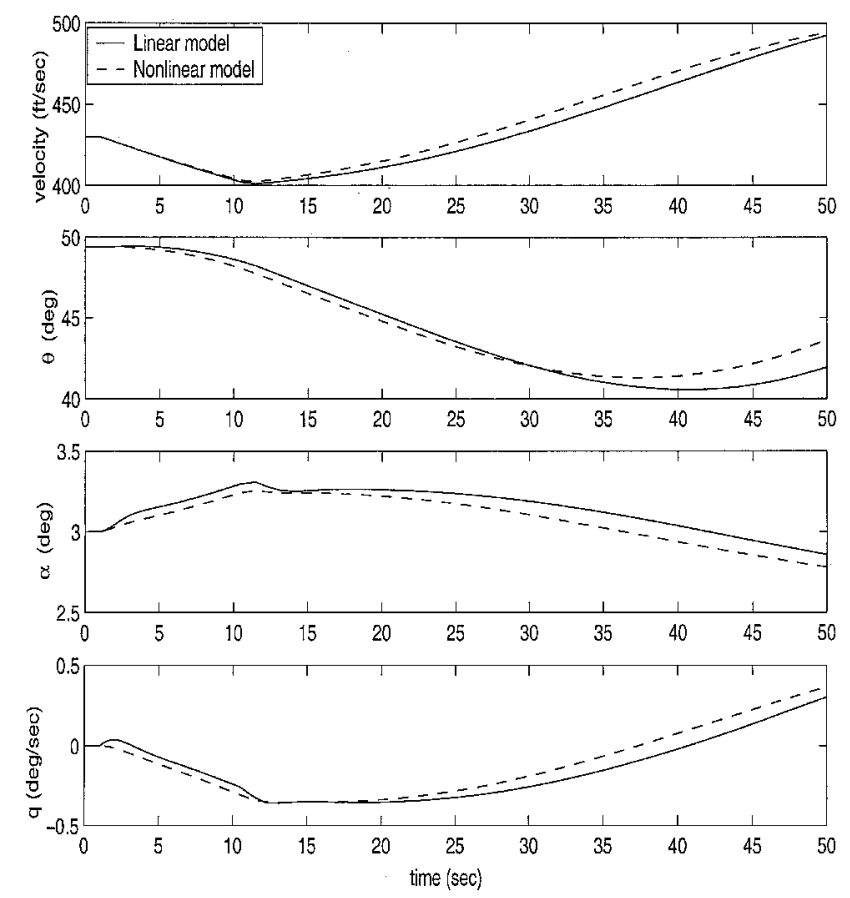

Fig. 1 Nonlinear vs quasi-LPV model simulations.

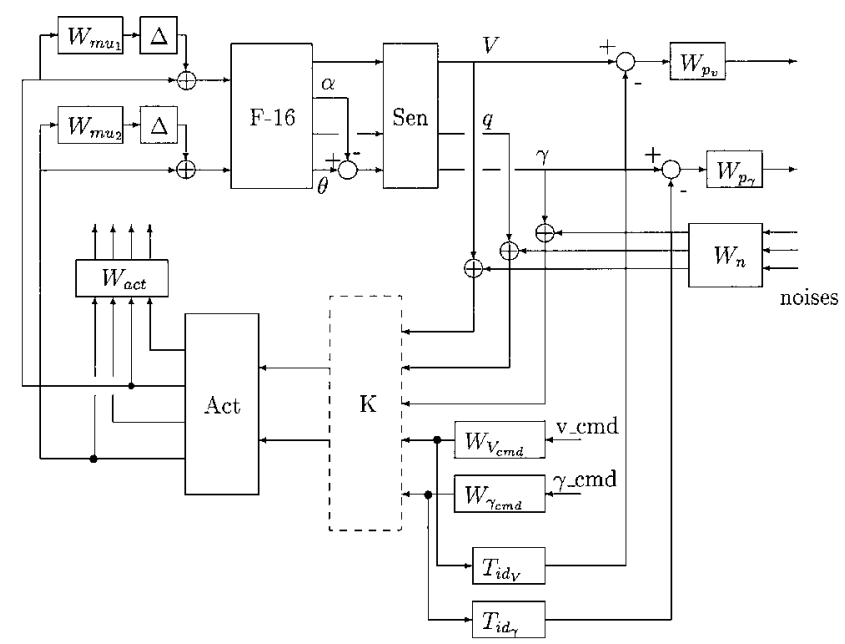

Fig. 2 Interconnection structure for the model matching problems.

\section{LPV Control Problem Formulation \\ Control Design Objectives and Weighting Functions}

A formulation of the LPV control synthesis of the F-16 longitudinal axes is presented in this section. The primary control objective for the F-16 longitudinal flight controller is to track velocity and flight-path angle commands within $1 \mathrm{ft} / \mathrm{s}$ and a 0.6 -deg error range in steady-state conditions.

Velocity and flight-path angle tracking problems can be formulated as model matching problems in the LPV control synthesis. In this paper, we consider the F-16 aircraft as an unmanned aircraft. The ideal transfer function from the flight-path angle command to the flight-path angle measurement is modeled as a second-order system, $0.426(s+1.5) /\left(s^{2}+1.6 s+0.64\right)$ with $0.8 \mathrm{rad} / \mathrm{s}$ bandwidth and a right-hand zero at $-1.5 \mathrm{rad} / \mathrm{s}$. For the velocity tracking problem, the ideal transfer function from the velocity command to the velocity measurement is modeled as the second-order system $0.16 /\left(s^{2}+0.8 s+0.16\right)$ with $0.4 \mathrm{rad} / \mathrm{s}$ natural frequency and critical damping.

A block diagram of the interconnectionstructure for synthesizing an LPV controller for the F-16 longitudinal axes is shown in Fig. 2. The velocity, angle rate, and angle sensors are modeled as the firstorder low pass filters $50 /(s+50), 60 /(s+60)$, and $10 /(s+10)$ (Refs. 16 and 17). In the LPV controller synthesis models, the sensor 
models shown in Fig. 2 are approximated as

$$
\text { sensor }=\operatorname{diag}[1,1,10 /(s+10)]
$$

because the interesting frequency range for LPV control synthesis is less than $10 \mathrm{rad} / \mathrm{s}$. The reduced-order sensor models help to reduce the overall state order of an LPV controller because the state order of an LPV controller is same as that of the augmented open-loop system. The elevator actuator is modeled as the first-order lag filter $20 /(s+20)$ and its rate limit is defined as $\pm 60 \mathrm{deg} / \mathrm{s}$ in Refs. 16 and 17 . Because the F-16 is a fighter aircraft, we estimated the engine model as the first-order lag filter $4 /(s+4)$, which allows fast responses in engine dynamics. The thrust rate limit is taken to be $10,000 \mathrm{lb} / \mathrm{s}$. In the block diagram in Fig. 2, the actuator rate and actuator models are

$$
\text { actuator }=\operatorname{diag}\left[\frac{20 s}{s+20}, \quad \frac{20}{s+20}, \frac{4 s}{s+4}, \frac{4}{s+4}\right]
$$

The performance weighting functions are chosen based on the desired performance objectives. The performance weighting function of flight-pathangle $W_{p_{\gamma}},\left[100(s / 100+1)^{2}\right] /(s / 0.6+1)^{2}$, is derived based on the performance objective to keep a $\gamma$ tracking error less than $0.6 \mathrm{deg}$ for a 1-rad command at steady-state flight. Because the bandwidth of the ideal model from the flight-path angle command to the flight-path angle measurement is $0.8 \mathrm{rad} / \mathrm{s}$, the roll-off frequency of the weighting function is chosen as $0.6 \mathrm{rad} / \mathrm{s}$ to specify the tracking error less than $0.6 \mathrm{deg}$ at the low-frequency region $(<0.6 \mathrm{rad} / \mathrm{s})$. The performance weighting function for the velocity $W_{p_{v}},(s / 200+1) /(s+1)$, is derived to track velocity commands within $1-\mathrm{ft} / \mathrm{s}$ error range in steady-state flight. The unmodeled dynamics are included in the multiplicative uncertainty models $W_{\mathrm{mu}_{1}}$ and $W_{\mathrm{mu}_{2}}$. The uncertainty weighting functions are rolled up in the midfrequency range to limit the bandwidth of the LPV controllers. The multiplicative uncertainty weight functions are set as

$$
W_{\mathrm{mu}_{1}}=\frac{0.01(s / 0.35+1)}{s / 80+1}, \quad W_{\mathrm{mu}_{2}}=\frac{0.01(s / 0.2+1)}{s / 50+1}
$$

The sensor noise models are taken as constant across frequency to reduce the state order of the LPV controllers. The velocity, angle, and angle rate sensornoises are modeled as white noises with amplitudes of $0.8 \mathrm{ft} / \mathrm{s}, 0.1 \mathrm{deg}$, and $0.6 \mathrm{deg} / \mathrm{s}$, respectively.

To solve LMI Eqs. (3-5), the basis function sets need to be defined for $X$ and $Y$. There is no analytical method to choose the best basis function set. Most often, the basis functions used are power series ${ }^{18}$ Legendre polynomials,${ }^{19}$ or affine functions of scheduling parameters. ${ }^{20}$ Here, the basis function set for $X(\rho)$ and $Y(\rho)$ is chosen as the first-order power series $\{1, \rho\}$ of the scheduling parameter of velocity to reduce the computation time in the LPV control synthesis. Note that the basis functions of $X$ and $Y$ do not have to be the same.

\section{Blending Two Controllers}

In this section, synthesizing an LPV controller for the F-16 longitudinal axes using the blending approach is demonstrated. To apply the blending approach for control of the F-16, the entire parameter set (the flight envelope) is partitioned into two subsets: high- and low-altitude regions. Parameter subsets are

$\mathcal{P}_{1} \equiv\{(V, \alpha, h) \mid 100 \leq V \leq 900 \mathrm{ft} / \mathrm{s},-10 \leq \alpha<45 \mathrm{deg}$,

$$
5000 \leq h<30,000 \mathrm{ft}\}
$$

$\mathcal{P}_{2} \equiv\{(V, \alpha, h) \mid 100 \leq V \leq 900 \mathrm{ft} / \mathrm{s},-10 \leq \alpha<45 \mathrm{deg}$,

$$
10,000<h \leq 40,000 \mathrm{ft}\}
$$

$\mathcal{P}_{\cap} \equiv \mathcal{P}_{1} \cap \mathcal{P}_{2}$

To use methods 1 and 2, blending functions are required over the parameter subset $\mathcal{P}_{\cap}$, which are satisfied with the boundary conditions of Eqs. (16) and (17). Blending functions $b_{1}(h)$ and $b_{2}(h)$ are chosen as $b_{1}(h)=$

$$
\begin{cases}1, & h \leq 10,000 \mathrm{ft} \\ 0.5\left[1+\cos \left(\frac{h-10,000}{20,000} \pi\right)\right], & 10,000<h<30,000 \mathrm{ft} \\ 0, & 30,000 \mathrm{ft} \leq h\end{cases}
$$

$b_{1}(h)+b_{2}(h)=1$

With the blending functions, the solution matrices $X_{i}$ and $Y_{i}$ are blended across the parameter subset $\mathcal{P}_{\cap}$ as

$$
\begin{gathered}
X_{b}(V, \alpha, h)=b_{1}(h) X_{1}(V, \alpha)+b_{2}(h) X_{2}(V, \alpha) \\
Y_{b}(V, \alpha, h)=b_{1}(h) Y_{1}(V, \alpha)+b_{2}(h) Y_{2}(V, \alpha)
\end{gathered}
$$

With methods 1 and 2, LPV controllers $K_{\mathrm{I}}$ and $K_{\mathrm{II}}$ are constructed with the solution matrices $X_{b}$ and $Y_{b}$ over the entire parameter set, respectively.

To blend solution matrices $X_{i}(V, \alpha)$ and $Y_{i}(V, \alpha)$ calculated over each parameter subset, the basis functions for blending matrix functions are required to use method 3 . In this paper, the basis function sets are chosen as $\left\{g_{0}, g_{1}\right\}$ for $X_{b_{1}}$ and $Y_{b_{1}}$, and as $\left\{h_{0}, h_{1}\right\}$ for $X_{b_{2}}$ and $Y_{b_{2}}$, respectively:

$g_{1}(h)=$

$$
\begin{cases}0, & h \leq 10,000 \mathrm{ft} \\ 0.15\left[1+\cos \left(\frac{h-20,000}{10,000} \pi\right)\right], & 10,000<h<30,000 \mathrm{ft} \\ 0, & 30,000 \mathrm{ft} \leq h\end{cases}
$$

$$
g_{0}(h)=b_{1}(h), \quad h_{0}(h)=1-g_{0}(h), \quad h_{1}(h)=-g_{1}(h)
$$

where $b_{1}(h)$ is defined in Eq. (45). Note that it is not necessary to choose the same basis functions for $X_{b_{k}}$ and $Y_{b_{k}}$. From the solution of the LMI optimization of Eq. (31), the matrices $X_{b}(V, \alpha, h)$ and $Y_{b}(V, \alpha, h)$ are calculated using Eqs. (26) and (29). An LPV controller $K_{\text {III }}$ is constructed from the matrices $X_{b}(V, \alpha, h)$ and $Y_{b}(V, \alpha, h)$ over the entire parameter set.

For comparison, an LPV controller $K_{\text {tot }}$ is constructed with solution matrices $X$ and $Y$ over the entire parameter set using the conventional LPV controller synthesis approach. It takes approximately $43 \mathrm{~h}$ on $933-\mathrm{MHz}$ Pentium III machine running Linux. With the blending methods 1,2 , and 3, it takes approximately 22,25 , and $30 \mathrm{~h}$ on the same machine, respectively. The computation time to synthesize the LPV controller for the F-16 longitudinal axes is reduced using the blending approaches.

\section{Nonlinear Simulations}

The 12 state nonlinear F-16 aircraft dynamics ${ }^{16}$ with the synthesized LPV controllers $K_{1}, K_{2}, K_{\text {tot }}, K_{\mathrm{I}}, K_{\mathrm{II}}$, and $K_{\text {III }}$ are simulated to compare their time simulations in this section. Recall that the controllers $K_{1}$ and $K_{2}$ are constructed over parameter subsets $\mathcal{P}_{1}$ and $\mathcal{P}_{2}$, respectively. The controller $K_{\text {tot }}$ is synthesized over the entire parameter set, using the conventional LPV control synthesis methodology. The controllers $K_{\mathrm{I}}, K_{\mathrm{II}}$, and $K_{\mathrm{III}}$ are the blended controller using the blending methods 1,2 , and 3 , respectively. In simulations, the LPV controlleris implemented using linear interpolation at the current values of the scheduling parameters between the grid point solutions. The full-state models of actuators and sensors are included in the nonlinear F-16 aircraft simulations.

With these controllers, the velocity and $\gamma$ step responses are simulated around 8000- and 32,000-ft altitude, respectively. The step input sizes are a $10-\mathrm{ft} / \mathrm{s}$ velocity command and a $5-\mathrm{deg} \gamma$ command at $1 \mathrm{~s}$. The simulation results in Fig. 3 show that all velocity and $\gamma$ measurements match the ideal responses within $\pm 0.25 \mathrm{ft} / \mathrm{s}$ and a 0.06-deg tracking error. It is observed that these controllers achieve the desired performance objectives.

The controllers $K_{1}, K_{\mathrm{I}}, K_{\mathrm{II}}$, and $K_{\mathrm{III}}$ are exactlyequal to each other over the parameter subset $\mathcal{P}_{1}-\mathcal{P}_{\cap}$ because the blending methods 1 , 


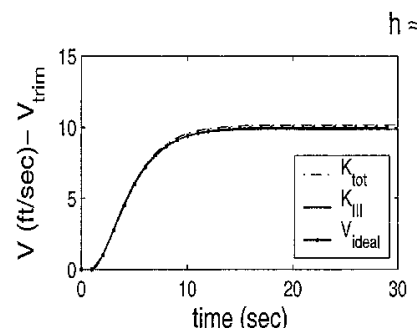

$h \approx 8000 \mathrm{ft}$
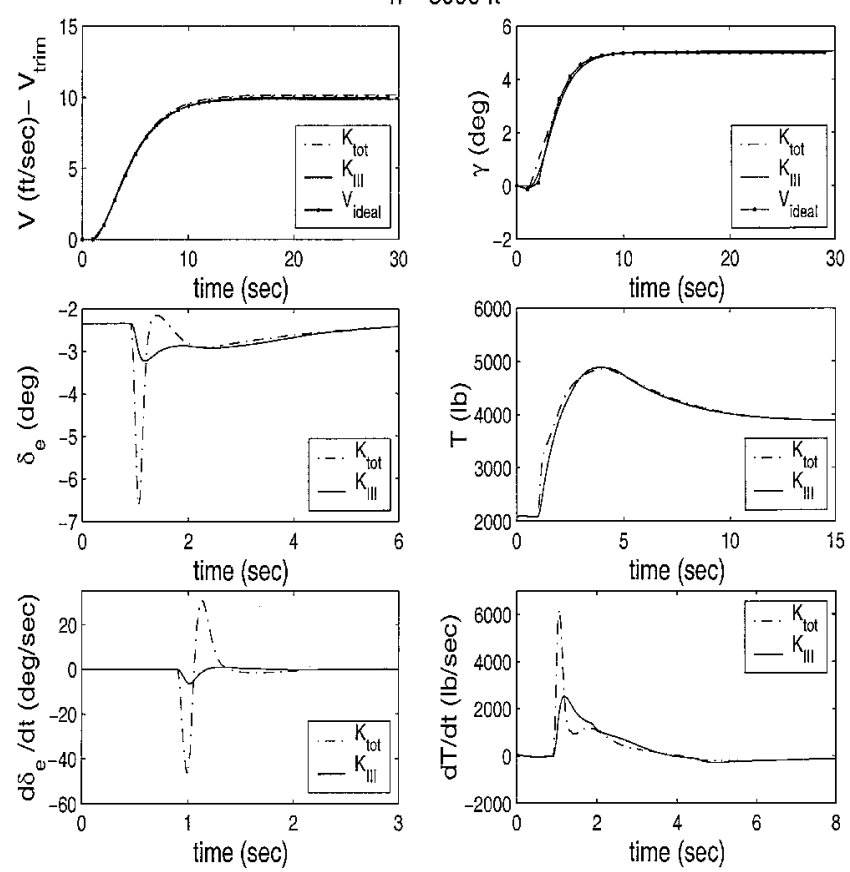

Fig. 3 Time simulations around 8000 -ft altitude.

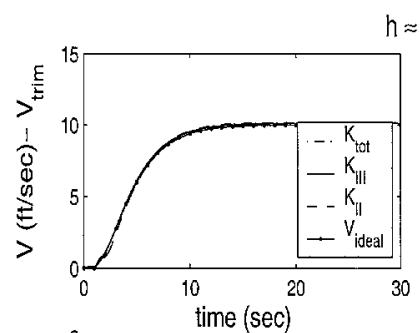

$h \approx 32000 \mathrm{H}$
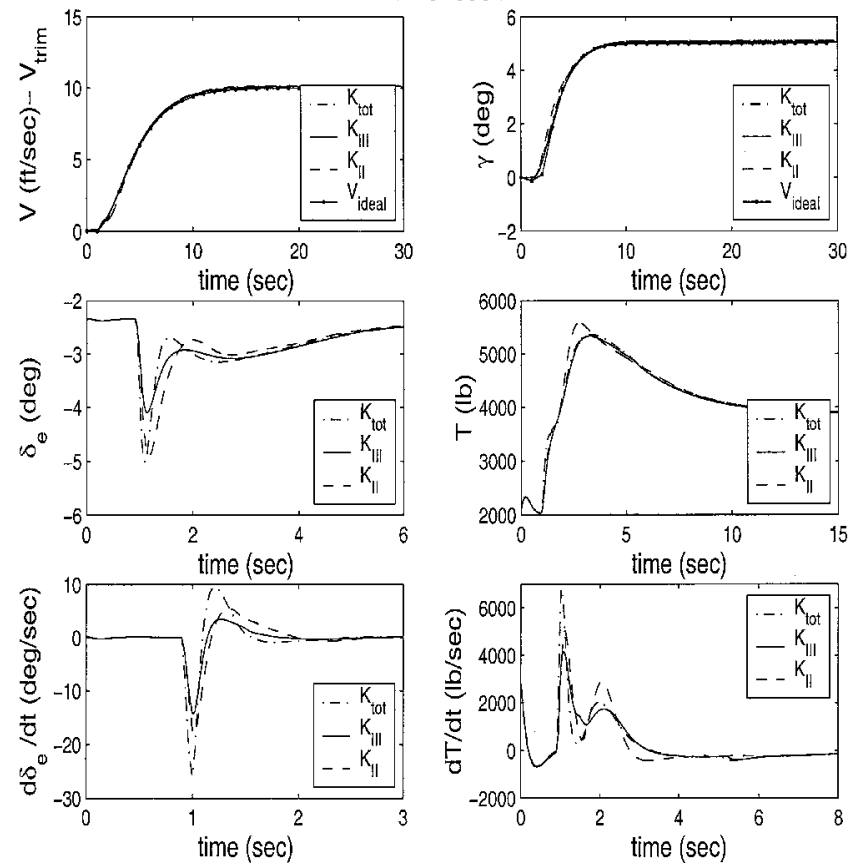

Fig. 4 Time simulations around 32,000-ft altitude.

2 , and 3 keep the regional controller $K_{1}$ over the parameter subset $\mathcal{P}_{1}-\mathcal{P}_{\cap}$. The step responses of velocity and $\gamma$ with the controller $K_{\text {III }}$ in Fig. 3 represent the step responses with the controllers $K_{1}$, $K_{\mathrm{I}}$, and $K_{\mathrm{II}}$ in the simulations around 8000 -ft altitude. The actuator deflections and their rates of the time simulations are shown in Fig. 3. Note that the blended controller $K_{\text {III }}$ uses smaller actuator deflections and their rates than the controller $K_{\text {tot }}$ does to achieve the performance objectives.

The controllers $K_{2}, K_{\mathrm{I}}$, and $K_{\mathrm{III}}$ are exactly equal to each other over the parameter subset $\mathcal{P}_{2}-\mathcal{P}_{\cap}$. The blended controller $K_{\text {II }}$ is different from $K_{2}$ over the parameter subset $\mathcal{P}_{2}-\mathcal{P}_{\cap}$ because in the method 2, the blended controller is constructed solving the LMI optimization of Eqs. (2-5) with the extra LMI constraints of Eqs. (24) and (25). The step responses with the controllers $K_{\text {tot }}, K_{\text {III }}$, and $K_{\text {II }}$ are shown in Fig. 4. It is observed that these LPV controllers achieve
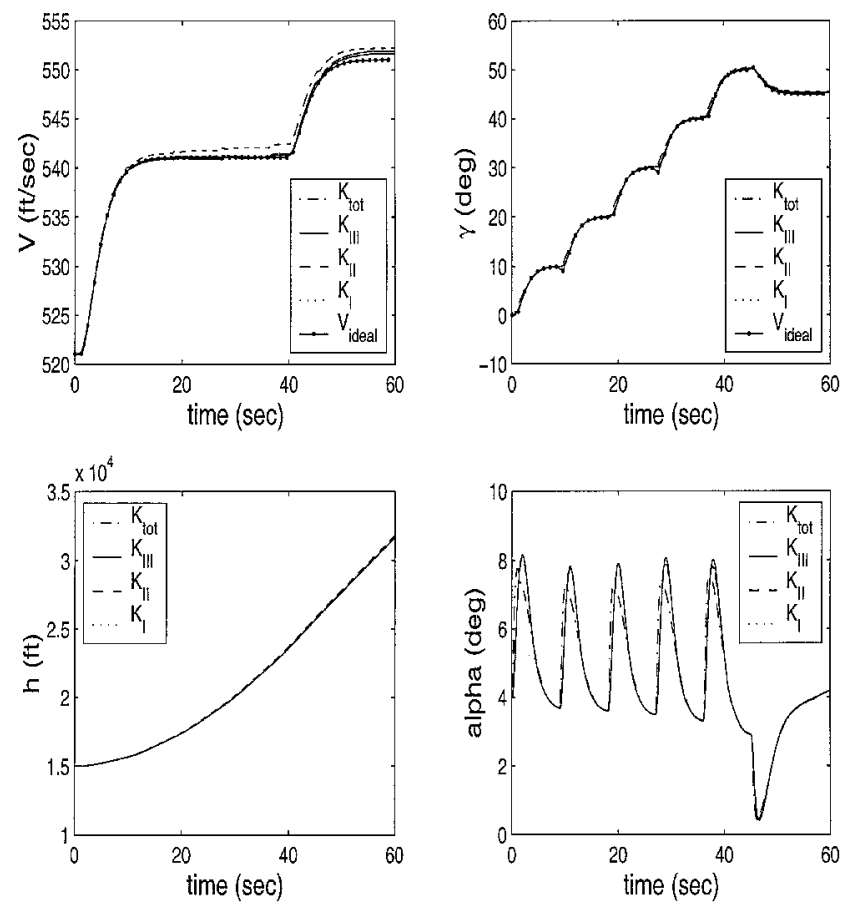

Fig. 5 Time simulations for the candidate maneuver.
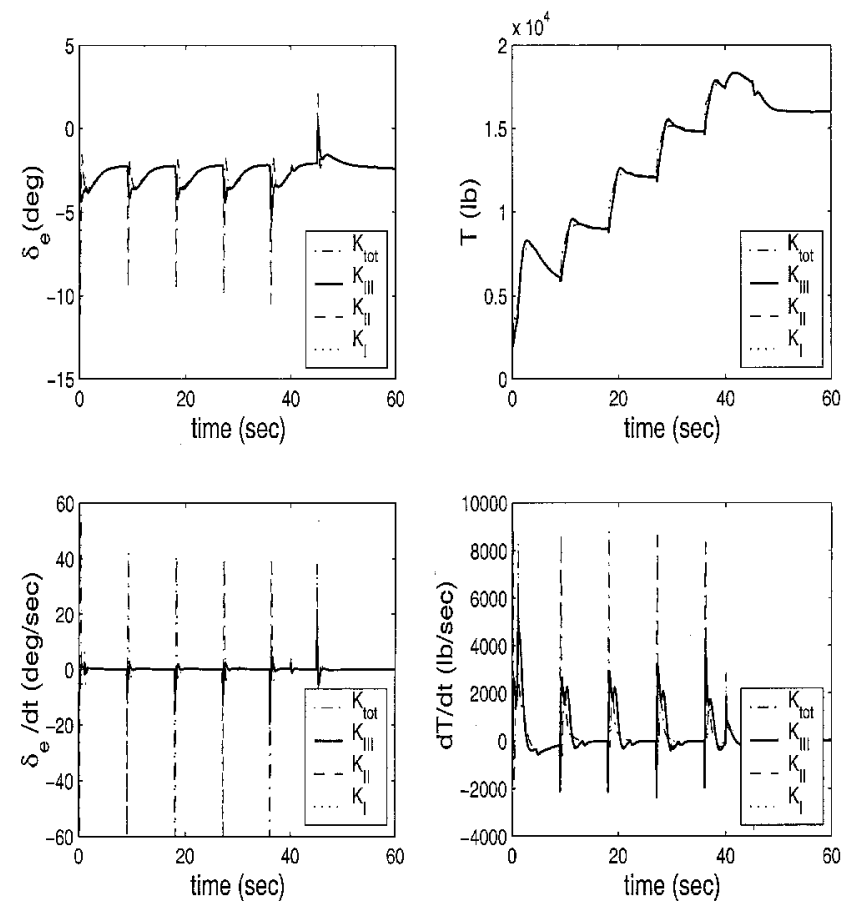

Fig. 6 Actuator time responses for the candidate maneuver.

the desired performance objectives over the parameter set $\mathcal{P}_{2}-\mathcal{P}_{\cap}$. The simulation results show that the controller $K_{\text {tot }}$ uses the largest actuator deflections and their rates to achieve the performance objectives.

For comparison, one of the candidate maneuvers that the F-16 aircraft flies from 15,000 to $32,000 \mathrm{ft}$ across the parameter subset $\mathcal{P}_{\cap}$ is simulated, using $20-$ and $10-\mathrm{ft} / \mathrm{s}$ velocity steps and $10-\mathrm{deg}$ $\gamma$ step commands. Velocity, flight-path angle, altitude, and angleof-attack time responses with the LPV controllers $K_{\text {tot }}, K_{\mathrm{III}}, K_{\mathrm{II}}$, and $K_{\mathrm{I}}$ are shown in Fig. 5. All of the LPV controllers can achieve the performance objectives across the parameter subset $\mathcal{P}_{\cap}$. Notice that the velocity tracking performance with the blended controllers is slightly better than the LPV controller $K_{\text {tot }}$ designed over the entire parameter set $\mathcal{P}_{1} \cup \mathcal{P}_{2}$ using the conventional LPV controller synthesis methodology. The actuator deflections and their rates with 
the LPV controllersare shown in Fig. 6. The simulation results show that the blended controllers use much smaller actuator deflections to track the velocity and $\gamma$ commands.

Sensor noises are integrated into the F-16 aircraft simulations for the same situation of flying the F-16 aircraft from 15,000 to $32,000 \mathrm{ft}$. Sensor noises for pitch rate, velocity, and pitch angle are set as white noises with $\pm 0.5 \mathrm{deg} / \mathrm{s}, \pm 0.8 \mathrm{ft} / \mathrm{s}$, and \pm 0.1 -deg amplitudes. The simulation results are omitted due to space limitations. The LPV controllers $K_{\text {tot }}, K_{\mathrm{III}}, K_{\mathrm{II}}$, and $K_{\mathrm{I}}$ stabilize the F-16 aircraft with the sensor noises and achieve tracking performance objectives over the scheduling parameter variations. The thrust signal and its rate do not exceed their limits: $T<19,000 \mathrm{lb}$ and $|\mathrm{d} T / \mathrm{d} t|<10,000 \mathrm{lb} / \mathrm{s}$. Also, the elevator actuator and its rates do not exceed their limits: $\left|\delta_{e}\right|<25 \mathrm{deg}$ and $\left|\mathrm{d} \delta_{e} / \mathrm{d} t\right|<60 \mathrm{deg} / \mathrm{s}$. Note that the blended controller can lead to a less conservative result than the conventional LPV controller because the blended controller can preserve the performance level of the regional LPV controllers.

Note that method 1 requires the slow parameter varying blending functions. In this example, the parameter intersection range is wide enough to provide the slow-varyingblending functionsfor method 1 . When the parameter intersection space is narrow, method 3 is appropriate to blend two LPV controllers over the parameter intersection space.

\section{Conclusions}

In this paper, the methodologies of blending LPV controllers are discussed to preserve the regionaloptimal solution over each parameter subset and to reduce computation time to synthesize an LPV controller over the entire parameter set. The blending approaches are to design each LPV controller for the set of a small number of scheduling parameters over the parameter subsets and blend all controllers scheduled on the entire scheduling parameters using the blending functions.

The quasi-LPV model of the F-16 longitudinalaxes is provided by a function substitutionmethod over the entire flight envelope to facilitate synthesis of an LPV controller. The two LPV controllers of the F-16 longitudinal axes are synthesized as functions of velocity and angle of attack at two regions: low and high altitudes, respectively. The two LPV controllers are blended into a single LPV controller as functions of velocity, angle of attack, and altitude over the entire flight envelope, using the three blending approaches. Note, from the nonlinear time simulations, that the blended controllers achieve better performance than the LPV controller constructed using the conventional LPV synthesis methodology. The blended controllers constructed using methods 1,2 , and 3 achieve the performance objectives and stabilize the closed-loop system of the F-16 aircraft with sensor noises.

\section{Appendix A: Nonlinear Equations of F-16 Longitudinal Axes}

From nonlinear equations (36-39), the nonlinear equations of the F-16 longitudinal axes are rewritten as follows:

$$
\left[\begin{array}{c}
\dot{V} \\
\dot{\alpha} \\
\dot{q} \\
\dot{\theta}
\end{array}\right]=\left[\begin{array}{ll}
A_{11}(V, \alpha) & A_{12}(V, \alpha, h) \\
A_{21}(V, \alpha) & A_{22}(V, \alpha, h)
\end{array}\right]\left[\begin{array}{c}
V \\
\alpha \\
q \\
\theta-\theta_{0}
\end{array}\right]
$$

$$
+M(V, \alpha, h) u_{s}+f(V, \alpha)
$$

where

$$
\begin{gathered}
A_{11}=A_{21}=\left[\begin{array}{ll}
0 & 0 \\
0 & 0
\end{array}\right] \\
A_{12}(1,1)=(\bar{q} S \bar{c} / 2 m V)\left[C_{X_{q}}(\alpha) \cos \alpha+C_{Z_{q}}(\alpha) \sin \alpha\right] \\
A_{12}(1,2)=g\left(-\cos \alpha \cos \theta_{0}-\sin \alpha \sin \theta_{0}\right) \\
A_{12}(2,1)=1+\left(\bar{q} S \bar{c} / 2 m V^{2}\right)\left[-C_{X_{q}}(\alpha) \sin \alpha+C_{Z_{q}}(\alpha) \cos \alpha\right] \\
A_{12}(2,2)=(g / V)\left(\sin \alpha \cos \theta_{0}-\cos \alpha \sin \theta_{0}\right)
\end{gathered}
$$

$$
A_{22}=\left\{\begin{array}{cc}
\left(\bar{q} S \bar{c} / 2 V I_{y y}\right)\left[\bar{c} C_{m_{q}}(\alpha)+\Delta C_{Z_{q}}(\alpha)\right] & 0 \\
1 & 0
\end{array}\right\}
$$

$$
f=\left[\begin{array}{c}
(\bar{q} S / m) C_{Z}(\alpha) \sin \alpha+g\left(-\cos \alpha \sin \theta_{0}+\sin \alpha \cos \theta_{0}\right) \\
(\bar{q} S / m V) C_{Z}(\alpha) \cos \alpha+(g / V)\left(\sin \alpha \sin \theta_{0}+\cos \alpha \cos \theta_{0}\right) \\
\left(\bar{q} S / I_{y y}\right) \Delta C_{Z}(\alpha) \\
0
\end{array}\right]
$$

The gain matrix $M$ is constructed as lookup tables and $u_{s}$ are synthetic inputs. The term of $M(V, \alpha, h) u_{s}$ can represent the following terms:

$$
\begin{aligned}
& M(V, \alpha, h) u_{s}\left(V, \alpha, \delta_{e}, T\right) \\
& \approx\left[\begin{array}{c}
(\bar{q} S / m)\left[\bar{C}_{Z_{e}} \delta_{e} \sin \alpha+C_{X}\left(\alpha, \delta_{e}\right) \cos \alpha\right]+(\cos \alpha / m) T \\
(\bar{q} S / m V)\left[\bar{C}_{Z_{e}} \delta_{e} \cos \alpha-C_{X}\left(\alpha, \delta_{e}\right) \sin \alpha\right]-(\sin \alpha / m V) T \\
\left(\bar{q} S \bar{c} / I_{y y}\right) C_{m_{0}}\left(\alpha, \delta_{e}\right)+\left(\bar{q} S / I_{y y}\right) \Delta \bar{C}_{Z_{e}} \delta_{e} \\
0
\end{array}\right]
\end{aligned}
$$

where $C_{Z}\left(\alpha, \delta_{e}\right)=C_{Z}(\alpha)+\bar{C}_{Z_{e}} \delta_{e}$. The aerodynamic coefficients data are taken from Ref. 16. The detailed methods to determine $M$ and synthetic inputs $u_{s}$ are available in Ref. 13. The synthetic inputs can vary in the range of $-1 \leq u_{1} \leq 1$ and $0 \leq u_{2} \leq 1$. The units of $u_{1}$ and $u_{2}$ are $25 \mathrm{deg}$ and $19,000 \mathrm{lb}$, respectively.

\section{Appendix B: Decomposition}

Set the state variables in a quasi-LPV model of the F-16 aircraft dynamics as $V-V_{0}, \alpha-\alpha_{0}, q-q_{0}$, and $\theta-\theta_{0}$, where $V_{0}, \alpha_{0}, q_{0}$ and $\theta_{0}$ represent a trim point. Then, Eq. (A1) is rewritten as

$$
\left[\begin{array}{l}
\dot{\xi}_{1} \\
\dot{\xi}_{2}
\end{array}\right]=F(z)+\left[\begin{array}{ll}
A_{11}(z) & A_{12}(z) \\
A_{21}(z) & A_{22}(z)
\end{array}\right]\left[\begin{array}{l}
\xi_{1} \\
\xi_{2}
\end{array}\right]+\left[\begin{array}{l}
B_{1}(z) \\
B_{2}(z)
\end{array}\right] \tilde{u}
$$

where

$$
\begin{gathered}
F(z)=f(z)+\left[\begin{array}{ll}
A_{11}(z) & A_{12}(z) \\
A_{21}(z) & A_{22}(z)
\end{array}\right]\left[\begin{array}{l}
z_{0} \\
w_{0}
\end{array}\right]+\left[\begin{array}{l}
B_{1}(z) \\
B_{2}(z)
\end{array}\right] u_{0} \\
\xi_{1}=z-z_{0}, \quad \xi_{2}=w-w_{0}, \quad \tilde{u}=u_{s}-u_{0} \\
z=\left[\begin{array}{ll}
V & \alpha
\end{array}\right]^{T}, \quad w=\left[\begin{array}{ll}
q & \theta
\end{array}\right]^{T}
\end{gathered}
$$

To provide a quasi-LPV model of F-16 aircraft dynamics from Eq. (B1), the term $F(z)$ should be decomposed into LPV functions written as

$$
F(z)=F\left(z_{0}+\xi_{1}\right)=\left[\begin{array}{c}
g_{z}\left(z_{0}+\xi_{1}\right) \\
g_{w}\left(z_{0}+\xi_{1}\right)
\end{array}\right] \xi_{1}
$$

where $g_{z} \in \mathcal{R}^{2 \times 2}$ and $g_{w} \in \mathcal{R}^{2 \times 2}$. There are an infinite number of possible solutions of $g_{z}$ and $g_{w}$ to satisfy Eq. (B4). To determine functions $g_{z}$ and $g_{w}$, more constraints are required. In this paper, the variation of $g_{z}$ and $g_{w}$ over the entire flight envelope is minimized. With these constraints, an optimization problem is formulated to determine $g_{z}$ and $g_{w}$. For example, to determine the first row of $g_{z}$, an optimization problem is as follows:

$$
\min _{g_{z_{11}} \in \mathcal{R}, g_{z_{12}} \in \mathcal{R}} \epsilon
$$

subject to

$$
\begin{gathered}
F_{1}\left(z_{0}+\xi_{1}\right)=\left[g_{z_{11}}\left(z_{0}+\xi_{1}\right) g_{z_{12}}\left(z_{0}+\xi_{1}\right)\right] \xi_{1} \\
\left|\frac{\partial^{2} g_{z_{11}}\left(z_{0}+\xi_{1}\right)}{\partial \xi_{1}^{2}}\right| \leq \epsilon, \quad\left|\frac{\partial^{2} g_{z_{12}}\left(z_{0}+\xi_{1}\right)}{\partial \xi_{1}^{2}}\right| \leq \epsilon
\end{gathered}
$$

where $F_{1}$ is the first row of $F$. To make the optimization problem of Eq. (B5) computationally tractable, the continuous constraints are evaluated at grid points over the parameter set. Thus, the matrix $g_{z}$ can be determined at every grid point of $\xi_{1}$. With solutions of the 
optimization for $g_{z}$ and $g_{w}$, a quasi-LPV model of F-16 longitudinal axes is written as

$$
\begin{aligned}
& {\left[\begin{array}{c}
\dot{V} \\
\dot{\alpha} \\
\dot{q} \\
\dot{\theta}
\end{array}\right]=\left[\begin{array}{ll}
A_{11}(V, \alpha)+g_{z}(V, \alpha) & A_{12}(V, \alpha, h) \\
A_{21}(V, \alpha)+g_{w}(V, \alpha) & A_{22}(V, \alpha, h)
\end{array}\right]\left[\begin{array}{c}
V-V_{0} \\
\alpha-\alpha_{0} \\
q \\
\theta-\theta_{0}
\end{array}\right]} \\
& \quad+M(V, \alpha, h)\left(u_{s}-u_{s 0}\right)
\end{aligned}
$$

\section{Acknowledgments}

This research was supported by Defense Advanced Research Projects Agency Software Enabled Control and NASA under NASA ContractNAS1-97046. The TechnicalMonitor was Christine Belcastro at NASA Langley Research Center.

\section{References}

${ }^{1}$ Shamma, J., and Athans, M., "Guaranteed Properties of Gain Scheduled Control for Linear Parameter-Varying Plants," Automatica, Vol. 35, No. 8, 1991, pp. 559-564.

${ }^{2}$ Shamma, J., and Athans, M., "Analysis of Gain Scheduled Control for Nonlinear Plants," IEEE Transactions on Automatic Control, Vol. 35, No. 8, 1990, pp. 898-907.

${ }^{3}$ Apkarian, P., and Adams, R., "Advanced Gain-Scheduling Techniques for Uncertain Systems," IEEE Transactions on Control Systems Technology, Vol. 6, No. 1, 1998, pp. 21-32.

${ }^{4}$ Becker, G., "Quadratic Stability and Performance of Linear Parameter Dependent Systems," Ph.D. Dissertation, Dept. of Mechanical Engineering, Univ. of California, Berkeley, CA, 1993.

${ }^{5}$ Wu, F., "Control of Linear Parameter Varying Systems," Ph.D. Dissertation, Dept. of Mechanical Engineering, Univ. of California, Berkeley, CA, 1995.

${ }^{6}$ Lee, L., "Identification and Robust Control of Linear Parameter-Varying Systems," Ph.D. Dissertation, Dept. of Mechanical Engineering, Univ. of California, Berkeley, CA, 1997.

${ }^{7}$ Wu, F., Packard, A., and Balas, G., "LPV Control Design for Pitch-Axis Missile Autopilots," Proceedings of the 34th IEEE Conference on Decision and Control, IEEE Publications, Piscataway, NJ, 1995, pp. 188-193.

${ }^{8}$ Shamma, J., "Gain-Scheduled Missile Autopilot Design Using Linear
Parameter-Varying Transformations," Journal of Guidance, Control, and Dynamics, Vol. 16, No. 2, 1993, pp. 256-261.

${ }^{9}$ Balas, G., Packard, A., Renfrow, J., Mullaney, C., and M'Closky, R., "Control of the F-14 Aircraft Lateral-Directional Axis During Powered Approach," Journal of Guidance, Control, and Dynamics, Vol. 21, No. 6, 1998, pp. 899-908.

${ }^{10}$ Balas, G., Fialho, I., Packard, A., Renfrow, J., and Mullaney, C., "On the Design of LPV Controllers for the F-14 Aircraft Lateral-Directional Axis During Powered Approach," Proceedings of the American Control Conference, American Automatic Control Council, Evanston, IL, 1997, pp. 123 127.

${ }^{11}$ Wolodkin, G., Balas, G., and Garrard, W., "Application to Parameter Dependent Robust Control Synthesis to Turbofan Engines," AIAA Paper 98-0973, 1998.

${ }^{12}$ Balas, G., Ryan, J., Shin, J.-Y., and Garrard, W., "New Technique for Design of Controllers for Turbofan Engines," AIAA Paper 98-3751, 1998.

${ }^{13}$ Shin, J.-Y., "Worst-Case Analysis and Linear Parameter-Varying GainScheduled Control of Aerospace Systems," Ph.D. Dissertation, Dept. of Aerospace Engineering and Mechanics, Univ. of Minnesota, Minneapolis, MN, Oct. 2000

${ }^{14}$ Tan, W., "Applications of Linear Parameter-Varying Control Theory," M.S. Thesis, Dept. of Mechanical Engineering, Univ. of California, Berkeley, CA, 1997.

${ }^{15}$ Tan, W., Packard, A., and Balas, G., "Quasi-LPV Modeling and LPV Control of a Generic Missile," Proceedings of the American Control Conference, American Automatic Control Council, Evanston, IL, 2000, pp. 3692 3696.

${ }^{16}$ Stevens, B., and Lewis, F., Aircraft Control and Simulation, Wiley, New York, 1992, pp. 584-592.

${ }^{17}$ Nguyen, L., Ogburn, M., Gilbert, W., Kibler, K., Brown, P., and Deal, P., "Simulator Study of Stall/Post-Stall Characteristics of a Fighter Airplane with Relaxed Longitudinal Static Stability," NASA TP 1538, 1979.

${ }^{18}$ Becker, G., "Parameter-Dependent Control of an Under-Actuated Mechanical System," Proceedings of the 34th IEEE Conference on Decision and Control, IEEE Publications, Piscataway, NJ, 1995, pp. 543-548.

${ }^{19}$ Primbs, J., "Nonlinear Optimal Control: A Receding Horizon Approach," Ph.D. Dissertation, Dept. of Engineering, Cambridge Univ., Cambridge, England, U.K., 1999.

${ }^{20}$ Wood, G., "Control of Parameter-Dependent Mechanical Systems," Ph.D. Dissertation, Dept. of Engineering, Cambridge Univ., Cambridge, England, U.K., 1995. 